\title{
Most-enhancing tumor volume by MRI radiomics predicts recurrence-free survival "early on" in neoadjuvant treatment of breast cancer
}

\author{
Karen Drukker ${ }^{*}$ D, Hui Li, Natalia Antropova, Alexandra Edwards, John Papaioannou and Maryellen L. Giger
}

\begin{abstract}
Background: The hypothesis of this study was that MRI-based radiomics has the ability to predict recurrence-free survival "early on" in breast cancer neoadjuvant chemotherapy.

Methods: A subset, based on availability, of the ACRIN 6657 dynamic contrast-enhanced MR images was used in which we analyzed images of all women imaged at pre-treatment baseline (141 women: 40 with a recurrence, 101 without) and all those imaged after completion of the first cycle of chemotherapy, i.e., at early treatment (143 women: 37 with a recurrence vs. 105 without). Our method was completely automated apart from manual localization of the approximate tumor center. The most enhancing tumor volume (METV) was automatically calculated for the pre-treatment and early treatment exams. Performance of METV in the task of predicting a recurrence was evaluated using ROC analysis. The association of recurrence-free survival with METV was assessed using a Cox regression model controlling for patient age, race, and hormone receptor status and evaluated by C-statistics. Kaplan-Meier analysis was used to estimate survival functions.

Results: The C-statistics for the association of METV with recurrence-free survival were 0.69 with 95\% confidence interval of $[0.58 ; 0.80]$ at pre-treatment and $0.72[0.60 ; 0.84]$ at early treatment. The hazard ratios calculated from Kaplan-Meier curves were 2.28 [1.08; 4.61], 3.43 [1.83; 6.75], and 4.81 [2.16; 10.72] for the lowest quartile, median quartile, and upper quartile cut-points for METV at early treatment, respectively.

Conclusion: The performance of the automatically-calculated METV rivaled that of a semi-manual model described for the ACRIN 6657 study (published C-statistic 0.72 [0.60; 0.84]), which involved the same dataset but required semimanual delineation of the functional tumor volume (FTV) and knowledge of the pre-surgical residual cancer burden.
\end{abstract}

Keywords: Breast cancer survival, Dynamic contrast-enhanced breast MRI, Most-enhancing tumor volume, Radiomics

\section{Background}

There is a large variation in the clinical presentation of, and outcome of, breast cancer in women. It has been shown that in many instances biological biomarkers, i.e., features, of the primary tumor correlate with outcome [1-5]. The availability of biomarkers that can be used to assess outcome as early and as accurately as possible is crucial to the development of successful targeted and personalized breast cancer therapies. Methods to assess such biological biomarkers for the prediction of outcome,

* Correspondence: kdrukker@uchicago.edu

Department of Radiology, MC2026, 5841 S Maryland Ave, Chicago, IL, USA however, may be invasive, expensive, not repeatable, or not widely available. Our hypothesis is that magnetic resonance (MR) image-based features obtained through quantitative image analysis will prove useful as noninvasive biomarkers for the assessment of, and prediction of, the response of breast cancer to neoadjuvant therapy. The goal of our research is to develop automatic and quantitative image-based surrogate biomarkers of breast cancer tumors for use in predicting recurrence and in association with recurrence-free survival, ultimately aiding in patient management. Moreover, our goal is to base predictions only on data available "early on" during patient 
treatment, i.e., data available pre-treatment and after the first cycle of neoadjuvant chemotherapy.

In the work presented here, we validated a single image-based biomarker for predicting recurrence and association with recurrence-free survival using MR images of breast tumors from the American College of Radiology Imaging Network (ACRIN) trial 6657, which was a multicenter study of contrast-enhanced MR imaging to assess breast tumor response to neoadjuvant chemotherapy [6]. A previous analysis of the ACRIN 6657 trial by Hylton et al. [7] showed that MR imaging was more strongly associated with pathologic response after neoadjuvant chemotherapy than clinical examination, with the greatest advantage measured early in treatment by using a volumetric measurement of tumor response. Hylton et al. [8] also demonstrated that the functional tumor volume, determined from dynamic contrast-enhanced MR images in a semi-manual manner, was predictive of recurrence-free survival. In this paper, we present our automatic and quantitative radiomics method for the determination of the most enhancing tumor volume as an image-based biomarker in the task of predicting recurrence and association with recurrence-free survival. The method proposed here is completely automated except for the manual localization of a single seedpoint in the approximate tumor center. Performance is assessed for our automated method - and compared to that for the functional tumor volume - for the prediction of recurrence, association with recurrence-free survival, and through Kaplan-Meier survival analysis.

\section{Methods}

\section{Dataset}

Dynamic contrast-enhanced magnetic resonance images (DCE-MRI) used in this work were obtained from a de-identified publicly available dataset and hence were IRB exempt for our study. The dataset has been described in detail elsewhere [8]. It was obtained from the ACRIN 6657 study and is available on The Cancer Imaging Archive $[9,10]$. Women with breast cancers measuring $3 \mathrm{~cm}$ or greater and who were scheduled to receive anthracycline-based neoadjuvant chemotherapy, were eligible for ACRIN 6657, and 237 women were accrued of whom 162 ultimately were enrolled (mean age 49 years, range 27-68 years). Women receiving non-anthracycline chemotherapy agents, such as trastuzumab, were excluded from ACRIN 6657. In our study, we used the images from the baseline exams obtained within 4 weeks before the start of chemotherapy, and those obtained at early treatment, which is defined as at least 2 weeks after the first cycle and before the second cycle of chemotherapy. Not all women (cases) were imaged at all treatment time points, resulting in a different number of exams being available at each treatment time point (Table 1). Of the 162 primary cancers, 78 were hormone-receptor positive (estrogen and/or progesterone receptor positive) and human epidermal growth factor receptor type 2 (HER2) negative, 41 were HER2 positive, 40 were triple negative, and for three the hormone receptor status was unknown.

Within the ACRIN 6657 study, recurrence-free survival was assessed for each patient based on clinical examination and mammography after surgery. The length of recurrence-free survival was defined as the time from initial surgery to local or distant recurrence or the time to last follow-up in patients without evidence of recurrence. We did not discriminate between women with local and distant recurrences, and as endpoint we used the length of recurrencefree survival.

MRIs were obtained using $1.5 \mathrm{~T}$ field-strength MR imaging systems [8] and we only used the dynamic contrast-enhanced series (gadolinium-based contrast agent). In-plane spatial resolution was $\leq 1 \mathrm{~mm}$ and slice thickness $\leq 2.5 \mathrm{~mm}$. All but one of the available exams had a single pre-contrast image and two images acquired at about $2 \frac{1}{2}$ minutes and $7 \frac{1}{1 / 2}$ minutes post contrastinjection, respectively (Table 1).

\section{Functional tumor volume (FTV)}

Apart from the MR images, the functional tumor volume was made available for most exams via the Cancer Imaging Archive (Table 1) [10]. The functional tumor volume (FTV) had been determined in a semiautomated fashion [11] using the signal enhancement ratio method [12]. Their method requires the manual placement of rectangular regions of interest (ROIs) on maximum intensity orthogonal projection images to delineate a 3D rectangular volumetric ROI that completely encloses a given tumor. Moreover, the method requires manual drawing of irregular ROIs to exclude enhancement regions within the $3 \mathrm{D}$ ROI that obviously do not correspond to the tumor if those are present (such as the heart or vessels) and manual adjustment of threshold values for the percent early enhancement and signal enhancement ratio, if so desired. Subsequently, the signal enhancement ratio - a measure combining contrast enhancement and washout - and percent early enhancement are calculated for each voxel in the 3D ROI and thresholded to obtain the FTV. For comparison to our methods and results, we used the published values for FTV that were provided with the images from the Cancer Imaging Archive and which were determined with a variable threshold for the percent early enhancement (default $70 \%$ but modified on a by-case basis as 
Table 1 Number of exams available (for a total of 162 women) at each treatment time point (with the number of cases with a recurrence vs.no recurrence in parentheses)

\begin{tabular}{|c|c|c|c|}
\hline & $\begin{array}{l}\text { Exams with at least } 3 \\
\text { acquisitions }\end{array}$ & $\begin{array}{l}\text { Functional Tumor } \\
\text { Volume }\end{array}$ & Both \\
\hline Pre-treatment baseline & 141 (40 vs. 101) & 137 (39 vs. 98) & 137 (39 vs. 98) \\
\hline Early treatment & 142 (37 vs. 105) & 143 (38 vs. 105) & 141 (37 vs. 104) \\
\hline Both at baseline and early treatment & 127 (37 vs. 90) & 124 (26 vs. 88) & 123 (36 vs. 87) \\
\hline
\end{tabular}

*For automated tumor segmentation, a dynamic contrast-enhanced MR exam needed at a minimum to consist of a pre-contrast and two post-contrast images; At baseline, all available exams met this criterion while at early treatment a single exam was eliminated from our automated analysis

needed) and zero threshold for the signal enhancement ratio [11].

\section{Most enhancing tumor volume (METV)}

In our method, for the calculation of the most enhancing tumor volume (METV), automated tumor segmentation and radiomics assessment required, at a minimum, the availability of a pre-contrast, first post-contrast, and second post-contrast image within an exam. As mentioned above, one of the exams (at early treatment) failed to meet this criterion (having only a single post-contrast image available) and was not analyzed. After placement, via manual localization, of a single seed-point on the approximate tumor center in only one of the slices within an exam, each tumor was automatically segmented in 3D by a fuzzy cmeans technique using the presence of contrast agent within each voxel over time within an automatically determined volume-of-interest centered at the manuallyindicated approximate lesion center [13]. This method is well-established and has been used extensively in its original form requiring identification of a region of interest as well as an updated version used in this work requiring only the manual indication of a seed-point [14-20]. In short, fuzzy c-means clustering is an unsupervised pattern recognition technique which was used to partition voxels into tumor and non-tumor groups. In order to do so, the kinetic curve was calculated for each voxel from the contrast enhancement over time including all available image acquisitions within a DCE-MRI sequence (both pre- and all postcontrast scans). In other words, this is a $4 \mathrm{D}$ segmentation method which for each case uses all 3D MR images within a DCE-MRI sequence. Voxels belonging to the surrounding parenchyma typically demonstrate limited contrast uptake over time while the voxels corresponding to a tumor demonstrate substantial contrast uptake early on in the DCE sequence followed by either a washout of contrast agent, a plateau in contrast uptake, or a continuous contrast uptake depending on tumor pathology. Using the kinetic curves for all voxels surrounding the manually-indicated approximate tumor center as input, fuzzy c-means partitioned these voxels into tumor and non-tumor groups in an unsupervised manner, i.e., without any manual determination of, or adjustment of, threshold values (as would be typical for the determination of FTV).

After the automated tumor segmentation, the most enhancing voxels within each segmented tumor were identified through a second fuzzy c-means clustering based on the kinetic curves of only voxels within the segmented tumor (Fig. 1) [21]. The voxels that demonstrated the most contrast enhancement within each tumor were identified by fuzzy c-means in an unsupervised manner without any manual determination of, or adjustment of, threshold values. The most enhancing tumor volume (METV) was defined as the aggregate volume of the identified most enhancing voxels. Note that the first fuzzy $\mathrm{C}$-means segmentation used as input an automatically-determined volume-of-interest surrounding each tumor (based on the seed-point location), while the input to the second segmentation step used only voxels within the computer-segmented tumor.

\section{Performance evaluation and statistical analysis}

We examined the values for METV and FTV at the baseline and early treatment exams for patients with and without a future recurrence through the use of box plots. Pearson correlation coefficients [22] were calculated to assess the relationship, if any, between METV and FTV.

For the baseline and early treatment time points, the association of METV and FTV with length of recurrence-free survival (in days) was assessed using a Cox regression model controlling for patient age, race, and hormone receptor status and evaluated by $\mathrm{C}$ statistics $[8,23,24]$. This model was made available by the organizers of the NCI Quantitative Imaging Network (QIN) Breast MRI Metrics of Response (BMMR) challenge.

For the early treatment time point, Kaplan-Meier curves [23, 24] for estimated recurrence-free survival were calculated and compared using a log-rank test using existing Matlab ${ }^{\mathrm{Tm}}$ code [25]. We compared recurrence-free survival estimates by using METV and FTV cut-points at the lowest (Q1), the median quartile (Q2), and the highest quartile (Q3). Mantel-Haenszel 


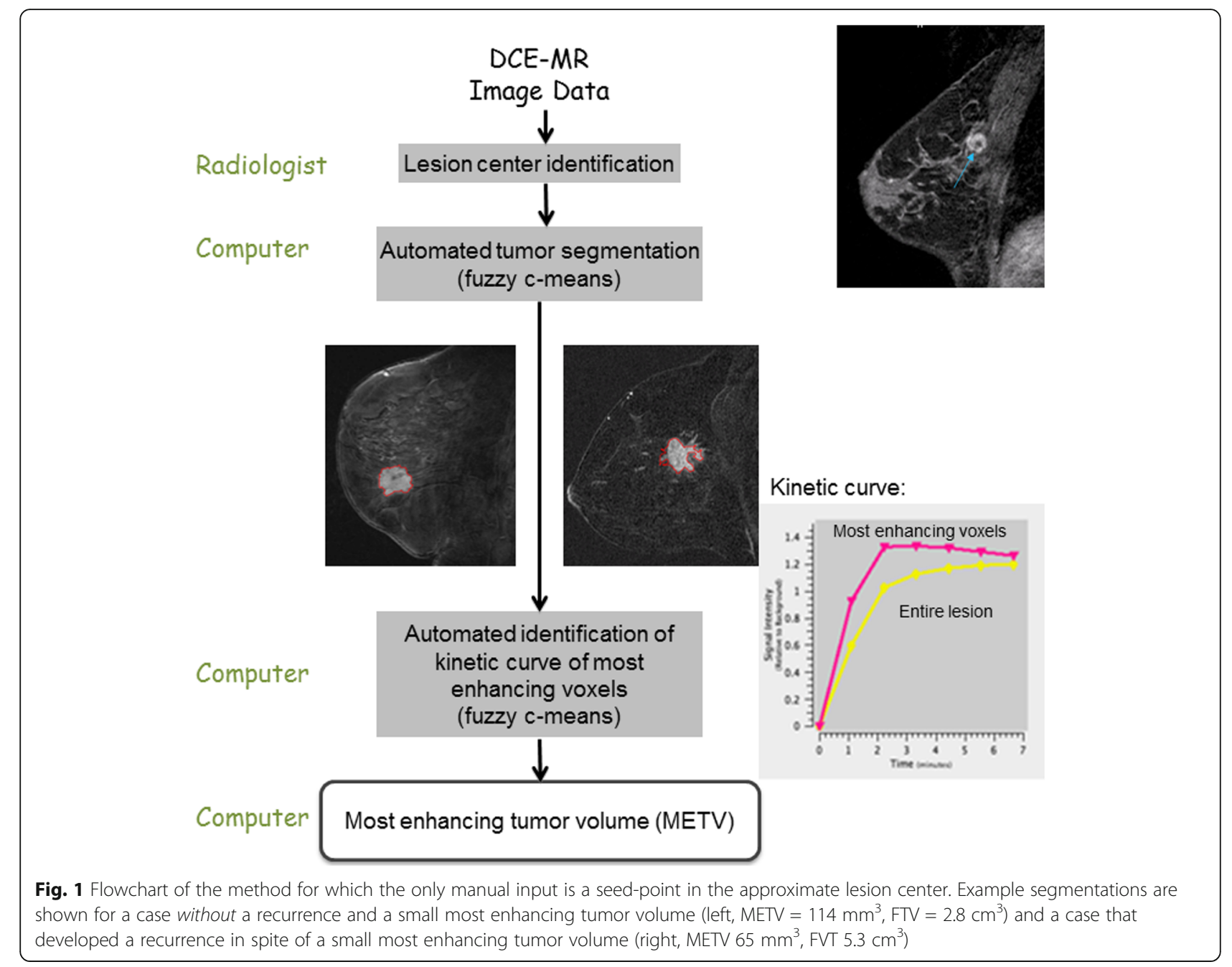

hazard ratios were calculated at the quartile cut-points for METV and for FTV. Non-inferiority was assessed in the task of predicting recurrence-free survival for METV compared to FTV at the early treatment time point by calculating the lower-bound of the one-sided $90 \%$ confidence interval for the difference in hazard ratio estimated through bootstrapping (1000 iterations). Kaplan-Meier survival curves were also estimated using the highest quartile cut-point (Q3) for METV at the early treatment time-point by hormone-receptor status subgroup, i.e., for hormone-receptor positive and HER2 negative, HER2 positive, and triple negative subgroups.

\section{Results}

METV was generally predictive of future recurrence with a high value indicative of a future recurrence and a low value indicative of the absence of recurrence. The ability of METV to predict a recurrence appeared to be similar to that for FTV [8] at the examined treatment time points (Fig. 2).
The correlation between METV and FTV measurements ranged from slight to substantial [26] when considering all available cases at baseline and early treatment (Table 2). Correlation appeared to be higher at the early treatment time point than at baseline, with correlation coefficients of $0.57\left(p=3.6 \cdot 10^{-12}\right)$ and 0.70 $\left(p=4.4 \cdot 10^{-19}\right)$, respectively (Table 2$)$. We found fair correlation between the changes in METV and FTV from baseline to early treatment with a correlation coefficient of $0.29\left(p=1.0 \cdot 10^{-3}\right)$.

In the association with length of recurrence-free survival, similar C-statistics were observed for METV and FTV. Both outperformed random guessing with their 95\% confidence intervals for the C-statistic excluding 0 . 5 , but the change in METV and FTV over time from pre-treatment to early treatment exams failed to do so (Table 3).

Kaplan-Meier plots comparing recurrence-free survival estimates for METV cut-points at the lowest quartile (Q1), the median quartile (Q2), and the highest quartile (Q3) at the early treatment time point all demonstrate 


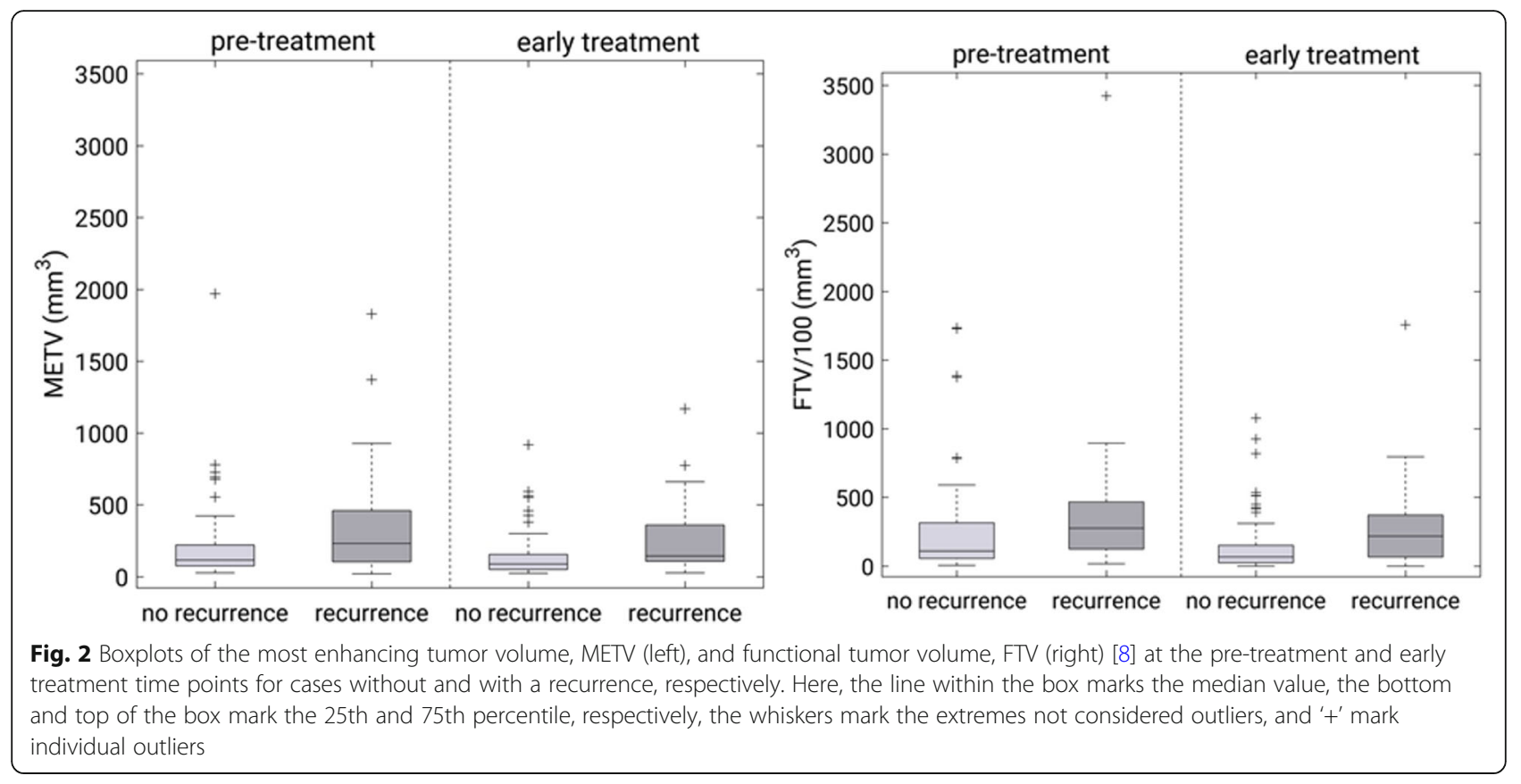

statistically significant differences with $p$-values in the log-rank test of 0.046, 0.001, and $<0.001$, respectively (Fig. 3). All The hazard ratios corresponding to the shown survival curves for METV (Fig. 3) and the equivalent for FTV demonstrate the potential for the use of either as a decision variable (Table 4). We failed to find any statistically significant differences between survival curves for METV and FTV and the corresponding hazard ratios (all $p$-values> 0.05). The lower bounds of the one-sided $90 \%$ confidence intervals for the differences in hazard ratios corresponding to cut points the lowest quartile, the median quartile, and the highest quartile (Table 4) for METV and FTV were $-1.53,-0.09$, and 6.14 , respectively, thus establishing, non-inferiority of METV to FTV only for the median cut point (Q2).

At the early treatment time point $(N=140$ cases with known hormone receptor status), comparison of the Kaplan-Meier survival curves obtained for the highest quartile (Q3) METV cut-point, demonstrate a statistically significant difference for hormone receptor-positive/HER2 negative $(N=66)$ and HER2 positive sub-groups $(N=38)$ with $p$-values in the log-rank test of 0.012 and 0.036 , respectively. For the triple negative cancers $(N=36)$ the comparison fails to demonstrate a statistically significant difference $(p=0.996)$ (Fig. 4).

\section{Discussion}

In this study we showed that the automaticallydetermined volume of the most enhancing region within a tumor measured on dynamic contrast-enhanced breast MRI, i.e., the most enhancing tumor volume (METV), was predictive of recurrence-free survival for breast cancer patients. Our method is straightforward to use in that it only requires the manual placement (localization) of a seed-point near the approximate tumor center; no bounding box, or variable manual thresholding is used. The method then automatically performs lesion segmentation and feature extraction in real time, making it a promising efficient tool for clinical application. Moreover, our work presented here only involved analysis of the pre-treatment and early treatment (acquired after the first cycle of neoadjuvant chemotherapy) MR exams.

In comparison to the functional tumor volume (FTV) measurements, which were publicly available for the

Table 2 Pearson correlation coefficients (with $p$-values) between the most enhancing tumor volume and functional tumor volume for the 123 cases for which both images and functional tumor volumes were available at baseline and at early treatment

\begin{tabular}{llll}
\hline & All $(N=123)$ & $\begin{array}{l}\text { Recurrence } \\
(N=36)\end{array}$ & $\begin{array}{l}\text { No recurrence } \\
(N=87)\end{array}$ \\
\hline Pre-treatment baseline & $0.57\left(3.6 \cdot 10^{-12}\right)$ & $0.34\left(4.0 \cdot 10^{-2}\right)$ & $0.78\left(5.7 \cdot 10^{-19}\right)$ \\
Early treatment & $0.70\left(4.4 \cdot 10^{-19}\right)$ & $0.69\left(3.6 \cdot 10^{-6}\right)$ & $0.63\left(7.1 \cdot 10^{-11}\right)$ \\
Delta (early treatment-baseline) & $0.29\left(1.0 \cdot 10^{-3}\right)$ & $-0.01\left(9.5 \cdot 10^{-1}\right)$ & $0.49\left(1.2 \cdot 10^{-6}\right)$ \\
\hline
\end{tabular}


Table 3 C-statistic (with 95\% confidence interval) for the association of recurrence-free survival (in days) using a Cox regression model controlling for patient age, race, and hormone receptor status

\begin{tabular}{lll}
\hline & C-statistic & \\
\cline { 2 - 3 } & $\begin{array}{l}\text { Most enhancing } \\
\text { tumor volume } \\
\text { METV (automated) }\end{array}$ & $\begin{array}{l}\text { Functional tumor } \\
\text { volume FTV } \\
\text { (semi-manual) }[8]\end{array}$ \\
\hline Pre-treatment baseline & $0.69[0.58 ; 0.80]$ & $0.67[0.55 ; 0.79]$ \\
Early treatment & $0.72[0.60 ; 0.84]$ & $0.70[0.58 ; 0.82]$ \\
Delta (early treatment-baseline) & $0.62[0.50 ; 0.75]$ & $0.64[0.50 ; 0.78]$ \\
\hline
\end{tabular}

dataset and previously published by Hylton et al. [8], it is interesting to note that the correlation between METV and FTV appeared to be higher at the early treatment time point than at baseline although at both treatment times the overall correlation was substantial [26]. The lower correlation at the pretreatment time point was caused by the low correlation between FTV and METV for patients with a recurrence. Upon closer investigation, however, this low correlation for patients with a recurrence was caused by a single outlier in FTV measurement (an extraordinarily high value for FTV). After removal of this single outlier, the correlation between FTV and METV at the pretreatment baseline was similar to that observed at the early treatment time point (correlation coefficients of $0.69\left(p=1.7 \cdot 10^{-18}\right)$ and 0.59 $\left(p=1.8 \cdot 10^{-4}\right)$, for the all patients and those with a recurrence, respectively).

In the work previously published by Hylton et al., using the same dataset [8], the "best" model for predicting recurrence-free survival involved both semi-manual determination of the functional tumor volume (FTV) and knowledge of the pre-surgical residual cancer burden (published C-statistic 0.72, 95\% confidence interval $[0.60 ; 0.84])$. A direct statistical comparison to that model was not possible but our best performing model including the most enhancing tumor volume at the early treatment exam, patient age, race, and hormone receptor status, achieved a C-statistic of $0.72[0.60 ; 0.84]$. Note that the residual cancer burden used in the "best" published model is determined post-surgery while our analyses (both for the most enhancing tumor volume and the functional tumor volume) included only information that is already available "early on" in patient treatment.

The performance of the change in volume from baseline to after the first cycle of chemotherapy, whether measured through METV or FTV, failed to have any predictive ability in the prediction of recurrence and association with recurrence-free survival. In other words, while both METV and FTV are predictors of recurrence-free survival, their change from baseline to the early treatment time point does not seem to be. This was also observed in the published ACRIN study, where FTV was shown to be a stronger predictor of recurrence -free survival than pathologic complete response [8]. In clinical practice, the change in tumor size is used an as indicator of treatment success. For size measurement, the response evaluation criteria in solid tumors (RECIST) refer to a set of published rules used to assess tumor burden in order to provide an objective assessment of response to therapy. They were initially introduced in 2000 and have undergone subsequent revision in 2009 [27]. In RECIST, for example, partial response is defined as a $30 \%$ decrease in the sum of all target lesions in longest axis measurement. So, while the change in tumor size is a clinically-used assessment of "short-term" response to therapy, the change in the functional tumor size does not seem to be a strong predictor of "longterm" treatment success in terms of recurrence-free survival [8].
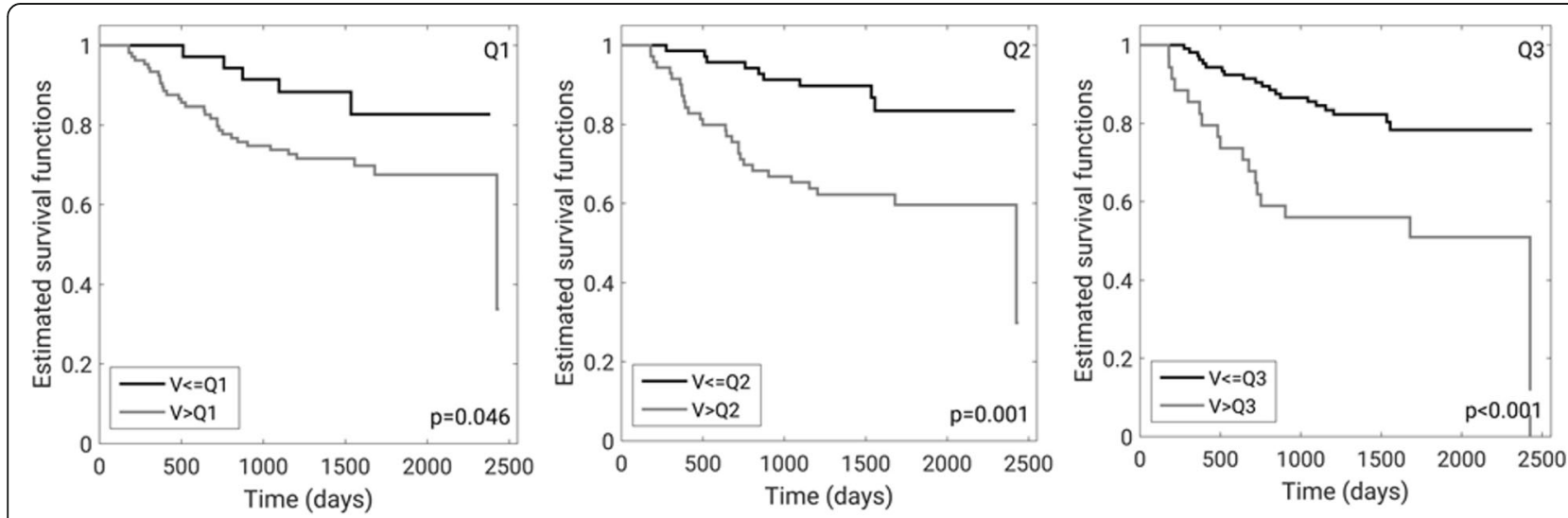

Fig. 3 Kaplan-Meier recurrence-free survival estimates for METV at the early treatment time point ( $N=142$ cases) using as cut-points the lowest quartile $(\mathrm{Q} 1$, left), median quartile $(\mathrm{Q} 2$, middle), and highest quartile $(\mathrm{Q} 3$, right) with corresponding $p$-values 
Table 4 Hazard ratios at early treatment for Kaplan-Meier curves using cut points at the lowest quartile (Q1), the median quartile (Q2), and the highest quartile (Q3) for METV and FTV (Fig. 3)

\begin{tabular}{lll}
\hline & $\begin{array}{l}\text { Most enhancing } \\
\text { tumor volume } \\
\text { METV (automated) }\end{array}$ & $\begin{array}{l}\text { Functional tumor } \\
\text { volume FTV } \\
\text { (semi-manual) }[8]\end{array}$ \\
\hline Lowest quartile Q1 & $2.28[1.08 ; 4.61]$ & $2.68[1.32 ; 5.48]$ \\
Median quartile Q2 & $3.43[1.83 ; 6.75]$ & $2.23[1.89 ; 4.31]$ \\
Highest quartile Q3 & $4.81[2.16 ; 10.72]$ & $5.31[2.59 ; 12.85]$ \\
\hline
\end{tabular}

The Kaplan-Meier curves for recurrence-free survival estimated from cut-points of METV at the early treatment time-point were similar to those for FTV published by Hylton et al. [8]. When recurrence-free survival was investigated by hormone receptor status subgroup, however, differences between the two approaches seem to become apparent. While using the highest quartile cut-point for FTV at the early treatment time-point the log-rank test yielded a statistically significant difference only for the triple-negative subgroup [8], performing the same analysis for METV yielded statistically significant differences for the hormone-receptor positive/HER2 negative, and HER2 positive subgroups while the difference for the triple negative subgroup failed to reach statistical significance (Fig. 4).

A limitation of this study was the modest size of the dataset. The ACRIN 6657 images and data have only recently become publicly available $[9,10]$, but the ACRIN 6657 protocol goes back to as far as late 2001. In the years elapsed since then, MR scanners have improved to provide better spatial resolution and better temporal resolution providing more numerous, and more closelyspaced in time, higher quality acquisitions in a dynamiccontrast enhanced MRI sequence. These factors will likely result in improved computerized analysis (for both the automated METV and probably also for the semimanual FTV). Another concern was that the ACRIN 6657 dataset was acquired before trastuzumab came into routine clinical use (for HER2 positive breast cancers), and that no patient contributing to the dataset was treated with trastuzumab or biosimilars. In order to continue our investigations into the prediction of response to therapy and recurrence-free survival, we are collecting a dataset at our own institution of pre- and posttreatment MR images of women with node-positive locally advanced breast cancers undergoing current clinical treatment protocols with approximately 70 cases collected to date. The ACRIN 6657 image data, however, remains very valuable since it was the result of a multiyear, multi-center clinical trial, which is very difficult to replicate at a single site.

\section{Conclusions}

Breast MR imaging provides prognostic information about tumor response already before any breast cancer treatment and after one cycle of chemotherapy that can potentially help guide treatment. Most enhancing tumor volume (METV), a quantitative radiomics feature calculated automatically in real time after placement of a seed-point on contrast-enhanced MR imaging, predicts recurrence-free survival for patients who receive neoadjuvant chemotherapy for breast cancer. METV predicts recurrence-free survival as early as pretreatment and after one cycle of standard anthracycline-based chemotherapy; in this study, METV measured pretreatment and after one cycle of chemotherapy, had comparable univariable Cstatistics (0.69, 95\% confidence interval $[0.58 ; 0.80]$ and 0.72 $[0.60 ; 0.84]$, respectively). Performance of METV in the prediction of recurrence-free survival appeared to be comparable to that for the functional tumor volume
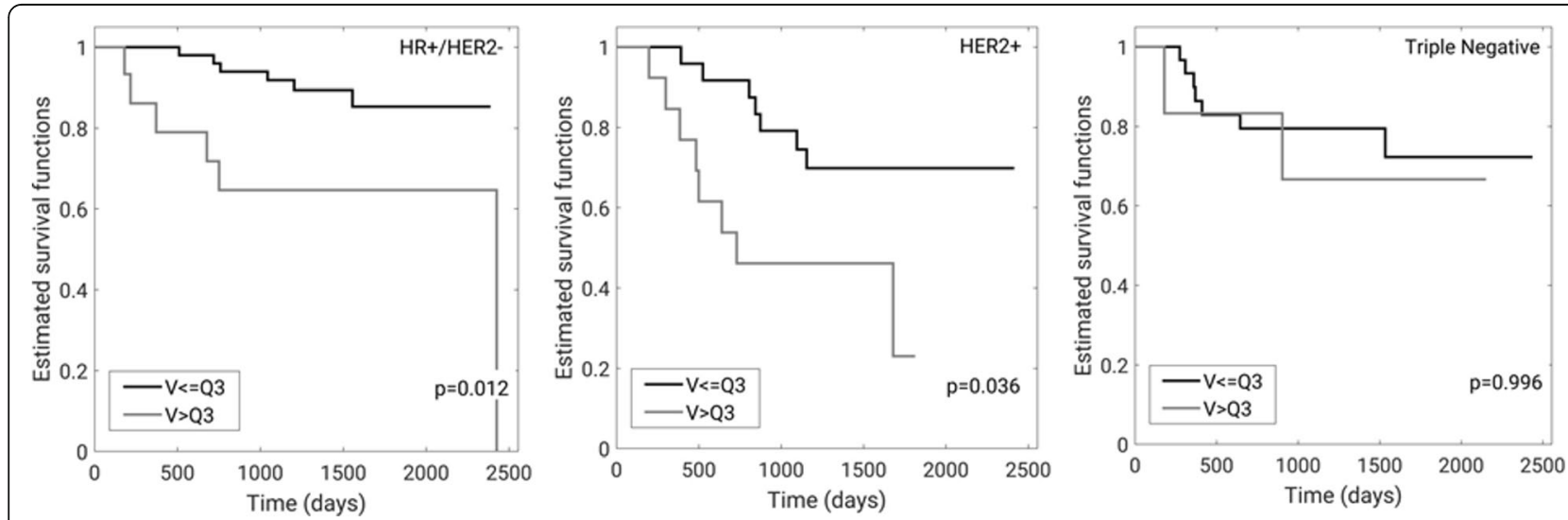

Fig. 4 Kaplan-Meier recurrence-free survival estimates for METV at the early treatment time point using the highest quartile cut-point (Q3) with corresponding p-values by hormone-receptor status subgroup: hormone-receptor positive and HER2 negative ( $N=66$, left), HER2 positive ( $N=38$, middle), and triple negative $(N=36$, right) with corresponding $p$-values (for 2 cases the hormone receptor status was unknown) 
(FTV), which is determined in a more semi-manual fashion requiring placement of a volumetric region of interest and, if so desired, manual adjustment of the threshold values for the percent early enhancement and signal enhancement ratio and/or manual exclusion of non-lesion regions of enhancement (univariable Cstatistic after one cycle of chemotherapy of 0.72 [0.60; 0.84] for METV versus $0.70[0.58 ; 0.82]$ for FTV, respectively).

In conclusion, the use of our automatic, computerextracted most enhancing tumor volume shows promise in the effective and efficient prediction of recurrence and in the association with recurrence-free survival. We investigated only the use of MR exams from "early on" in patient breast cancer neoadjuvant chemotherapy treatment, using only MRI exams acquired at the pretreatment baseline and after the first cycle of chemotherapy, thus potentially enabling changes to therapy well before excision.

\section{Abbreviations \\ ACRIN: American College of Radiology Imaging Network; FTV: Functional tumor volume; METV: Most enhancing tumor volume; QIN: Quantitative Imaging Network; RECIST: Response evaluation criteria in solid tumors; ROl: Region of interest}

\section{Acknowledgments}

N/A

\section{Funding}

U01CA195564, no involvement in study design, analysis, writing of manuscript, etc

\section{Availability of data and materials}

The image dataset is available at the Cancer Imaging Archive, 'Newitt D, Hylton N: on behalf of the I-SPY 1 Network and ACRIN 6657 Trial Team. (2016). Multi-center breast DCE-MRI data and segmentations from patients in the I-SPY 1/ACRIN 6657 trials. In: The cancer imaging archive. 2016.', https:// wiki.cancerimagingarchive.net/display/Public/ISPY1

\section{Authors' contributions}

$\mathrm{KD}$ was involved in study design; performed analysis of the image-based biomarkers for the prediction of recurrence-free survival; performed statistical analysis; was the main writer of the manuscript. $\mathrm{HL}$ assisted in analysis of image-based biomarkers. AE performed extraction of the image-based biomarkers. JP assisted in biomarker extraction and data acquisition. MLG was main designer of the study. All authors were involved in the writing of the manuscript and all read and approved the final version. All authors agree to be accountable for all aspects of the work.

\section{Ethics approval and consent to participate}

We used a publicly available anonymized dataset; hence IRB for this study was waived.

\section{Consent for publication}

N/A

\section{Competing interests}

KD receives royalties from Hologic. AE and JP are consultants to QView. MLG is a stockholder in Hologic Inc. and Quantitative Insights Inc., is co-founder of Quantitative Insights Inc., and receives royalties from Hologic Inc., General Electric Company, MEDIAN Technologies, Riverain Technologies LLC, Mitsubishi Corporation and Toshiba Corporation.

\section{Publisher's Note}

Springer Nature remains neutral with regard to jurisdictional claims in published maps and institutional affiliations.

Received: 10 January 2018 Accepted: 27 March 2018

Published online: 13 April 2018

\section{References}

1. Adamo B, Rita Ricciardi GR, leni A, Franchina T, Fazzari C, Sanò MV, Angelico G, Michele C, Tuccari G, Adamo V. The prognostic significance of combined androgen receptor, E-cadherin, Ki67 and CK5/6 expression in patients with triple negative breast cancer. Oncotarget. 2017;8(44):76974-86.

2. Criscitiello C, Bagnardi V, Pruneri G, Vingiani A, Esposito A, Rotmensz N, Curigliano G. Prognostic value of tumour-infiltrating lymphocytes in small HER2-positive breast cancer. Eur J Cancer. 2017;87:164-71.

3. Gupta I, Ouhtit A, Al-Ajmi A, Rizvi SGA, Al-Riyami H, Al-Riyami M, Tamimi Y. BRIP1 overexpression is correlated with clinical features and survival outcome of luminal breast cancer subtypes. Endocr Connect. 2017;

4. Kjaer IM, Bechmann T, Brandslund I, Madsen JS. Prognostic and predictive value of EGFR and EGFR-ligands in blood of breast cancer patients: a systematic review. Clin Chem Lab Med. 2017;

5. Niméus E, Folkesson E, Nodin B, Hartman L, Klintman M. Androgen receptor in stage I-II primary breast cancer-prognostic value and distribution in subgroups. Anticancer Res. 2017:37(12):6845-53.

6. Esserman LJ, Berry DA, Cheang MC, Yau C, Perou CM, Carey L, DeMichele A, Gray JW, Conway-Dorsey K, Lenburg ME, et al. Chemotherapy response and recurrence-free survival in neoadjuvant breast cancer depends on biomarker profiles: results from the I-SPY 1 TRIAL (CALGB 150007/150012; ACRIN 6657). Breast Cancer Res Treat. 2012;132(3):1049-62.

7. Hylton NM, Blume JD, Bernreuter WK, Pisano ED, Rosen MA, Morris EA, Weatherall PT, Lehman CD, Newstead GM, Polin S, et al. Locally advanced breast cancer: MR imaging for prediction of response to neoadjuvant chemotherapy-results from ACRIN 6657/I-SPY TRIAL. Radiology. 2012;263(3):663-72

8. Hylton NM, Gatsonis CA, Rosen MA, Lehman CD, Newitt DC, Partridge SC, Bernreuter WK, Pisano ED, Morris EA, Weatherall PT, et al. Neoadjuvant chemotherapy for breast cancer: functional tumor volume by MR imaging predicts recurrence-free survival-results from the ACRIN 6657/CALGB 150007 I-SPY 1 TRIAL. Radiology. 2016:279(1):44-55.

9. Newitt D, Hylton N, on behalf of the I-SPY 1 Network and ACRIN 6657 Trial Team. Multi-center breast DCE-MRI data and segmentations from patients in the I-SPY 1/ACRIN 6657 trials. In: The cancer imaging archive; 2016.

10. Clark K, Vendt B, Smith K, Freymann J, Kirby J, Koppel P, Moore S, Phillips S, Maffitt $D$, Pringle $M$, et al. The cancer imaging archive (TCIA): maintaining and operating a public information repository. J Digit Imaging. 2013;26(6): $1045-57$.

11. Jafri NF, Newitt DC, Kornak J, Esserman LJ, Joe BN, Hylton NM. Optimized breast MRI functional tumor volume as a biomarker of recurrence-free survival following neoadjuvant chemotherapy. J Magn Reson Imaging. 2014:40(2):476-82

12. Hylton NM. Vascularity assessment of breast lesions with gadoliniumenhanced MR imaging. Magn Reson Imaging Clin N Am. 1999;7(2):411-20

13. Chen W, Giger ML, Bick U. A fuzzy c-means (FCM)-based approach for computerized segmentation of breast lesions in dynamic contrast-enhanced MR images. Acad Radiol. 2006;13(1):63-72.

14. Burnside ES, Drukker K, Li H, Bonaccio E, Zuley M, Ganott M, Net JM, Sutton EJ, Brandt KR, Whitman GJ, et al. Using computer-extracted image phenotypes from tumors on breast magnetic resonance imaging to predict breast cancer pathologic stage. Cancer. 2015;

15. Zhu Y, Li H, Guo W, Drukker K, Lan L, Giger ML, Ji Y. Deciphering genomic underpinnings of quantitative MRI-based radiomic phenotypes of invasive breast carcinoma. Sci Rep. 2015:5:17787.

16. Schacht DV, Drukker K, Pak I, Abe H, Giger ML: Using quantitative image analysis to classify axillary lymph nodes on breast MRI: A new application for the Z 0011 Era. In: Eur J Radiol. Volume 41, edn. United States: Published by Elsevier Ireland Ltd.; 2014: 031915.

17. Bhooshan N, Giger M, Lan L, Li H, Marquez A, Shimauchi A, Newstead GM. Combined use of T2-weighted MRI and T1-weighted dynamic contrastenhanced MRI in the automated analysis of breast lesions. Magn Reson Med. 2011;66(2):555-64. 
18. Bhooshan N, Giger M, Edwards D, Yuan Y, Jansen S, Li H, Lan L, Sattar H, Newstead G. Computerized three-class classification of MRI-based prognostic markers for breast cancer. Phys Med Biol. 2011;56(18):5995-6008.

19. Chen W, Giger ML, Newstead GM, Bick U, Jansen SA, Li H, Lan L.

Computerized assessment of breast lesion malignancy using DCE-MRI robustness study on two independent clinical datasets from two manufacturers. Acad Radiol. 2010;17(7):822-9.

20. Chen W, Giger ML, Li H, Bick U, Newstead GM. Volumetric texture analysis of breast lesions on contrast-enhanced magnetic resonance images. Magn Reson Med. 2007;58(3):562-71.

21. Chen W, Giger ML, Bick U, Newstead GM. Automatic identification and classification of characteristic kinetic curves of breast lesions on DCE-MRI. Med Phys. 2006;33(8):2878-87.

22. Glantz SA. Primer of biostatistics. 5th ed. New York: McGraw-Hill, Medical Pub. Div; 2002.

23. Bland J, Altman D. Statistics notes - survival probabilities (the Kaplan-Meier method). Br Med J. 1998;317(7172):1572-2.

24. Altman D, Destavola B, Love S, Stepniewska K. Review of survival analyses published in cancer journals. Br J Cancer. 1995;72(2):511-8.

25. Cardillo G: LogRank: comparing survival curves of two groups using the log rank test. http://www.mathworks.com/matlabcentral/fileexchange/22317; 2008.

26. Landis J, Koch G. Measurement of observer agreement for categorical data. Biometrics. 1977;33(1):159-74.

27. Eisenhauer EA, Therasse P, Bogaerts J, Schwartz LH, Sargent D, Ford R, Dancey J, Arbuck S, Gwyther S, Mooney M, et al. New response evaluation criteria in solid tumours: revised RECIST quideline (version 1.1). Eur I Cancer. 2009;45(2):228-47.

\section{Submit your next manuscript to BioMed Central and we will help you at every step:}

- We accept pre-submission inquiries

- Our selector tool helps you to find the most relevant journal

- We provide round the clock customer support

- Convenient online submission

- Thorough peer review

- Inclusion in PubMed and all major indexing services

- Maximum visibility for your research

Submit your manuscript at www.biomedcentral.com/submit 